\title{
Aortic Pressure Waveforms Reconstruction Using Simplified Kalman Filter
}

\author{
Wenyan Liu ${ }^{1}$, Zongpeng $\mathrm{Li}^{1}$, Yang Yao ${ }^{1}$, Shuran Zhou ${ }^{1}$, Yuelan Zhang ${ }^{2}$, Lisheng $\mathrm{Xu}^{1}{ }^{*}{ }^{*}$ \\ ${ }^{1}$ Biomedical and Information Engineering, Northeastern University, Shenyang City, Liaoning \\ Province, China \\ ${ }^{2}$ The First Hospital of China Medical University, Shenyang City, Liaoning Province, China \\ ${ }^{3}$ Neusoft Research of Intelligent Healthcare Technology, Co. Ltd., Shenyang, China
}

\begin{abstract}
Aortic pressure $\left(P_{a}\right)$ waveforms are important for diagnosis of cardiovascular disease. However, the direct measurement of $P_{a}$ is invasive and expensive. In the paper, a new simplified Kalman filter (SKF) algorithm for blind system identification was employed for the reconstruction of $P_{a}$ waveforms using two peripheral artery pressure waveforms. The data of $P_{a}$ waveforms are collected from 24 human subjects. Simultaneously, brachial artery and femoral artery pressure waveforms data are generated from the simulation of a known two-channel finite impulse response system. In order to study the performance of the proposed SKF algorithm, different amounts of signal-tonoise ratio of the output signal were used in the experiment. Experimental results demonstrated that the proposed SKF algorithm had advantages in comparison with the canonical correlation analysis (CCA) algorithm. It is notable that the proposed SKF algorithm works much more noise-robust than the CCA algorithm in a wide range of $S N R$.
\end{abstract}

\section{Introduction}

Aortic pressure $\left(P_{a}\right)$ waveforms are a significant predictor of cardiovascular system risk. Left ventricular blood ejected is propagated to the body through the arterial tree of human and $P_{a}$ is formed at the aortic root. $P_{a}$ waveforms contain information of cardiac ejection and the arterial system properties [1]. However, the "gold standard" of $P_{a}$ measurement is invasive by cardiac catheterization which hasn't been widely used in clinical practice due to its risk and expensive cost. Therefore, methods of noninvasive measurement are important for obtaining $P_{a}$. These methods mainly include substitution methods and transfer function methods. Peripheral artery pressure $\left(P_{p}\right)$ waveforms are generally easier to be measured than $P_{a}$ waveforms. Usually brachial artery pressure $\left(P_{b}\right)$ waveforms are regarded as $P_{a}$ waveforms for surrogate.
However, the more accurate information of $P_{a}$ waveforms, such as systolic pressure and pulse pressure of $P_{a}$ waveforms may not be directly derived from $P_{p}$ waveforms [2]. Several mathematical methods are recently developed to estimate $P_{a}$ waveforms from noninvasively measured $P_{p}$ waveforms. These techniques have applied an average transfer function derived over a group of subjects to measured $P_{p}$ waveforms from other subjects to estimate the corresponding $P_{a}$ waveforms. However, transfer function methods need to use parameter values derived from prior experimentation [3]. Thus, it has attracted concern on noninvasive estimation method using $P_{p}$ waveforms to estimate $P_{a}$ waveforms in recent years [4, 5]. More recently, multichannel blind system identification (MBSI) algorithms have been proposed, such as the cross relation (CR) algorithm, the two-step maximum likelihood (TSML) algorithm, the subspace (SS) algorithm, the canonical correlation analysis (CCA) algorithm and so on. These methods can estimate $P_{a}$ waveforms satisfactorily when the signal-to-noise ratio (SNR) of observed signal is high [9]. In this paper, the proposed simplified Kalman filter (SKF) algorithm is capable of adaptive dynamic reconstruction $P_{a}$ waveforms to solve the transfer function based the signal with low SNR.

\section{Method}

Figure 1 illustrates the single-input two-output model of an arterial tree.

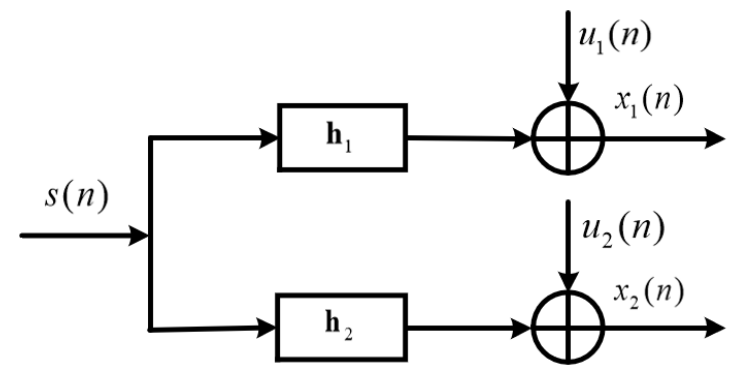

Figure 1. A block structure of two-channel FIR system. 
The proposed SKF of MBSI algorithms was applied to two peripheral artery pressure waveforms for reconstructing $P_{a}$ waveforms. As shown in Figure 1, a twochannel FIR system is presented. $s(n)$ denotes the input signal. $\mathbf{h}_{i}=\left[h_{i}(0), h_{i}(1), \cdots, h_{i}(L-1)\right]^{T}(i=1,2)$ denotes the impulse response of $i$-th unknown channel. $L$ is the maximal channel length. $u_{i}(n)$ is the additive noise. The output signal $x_{i}(n)$, as the result of a linear convolution between the input signal $s(n)$ and the corresponding channel impulse response $h_{i}(n)$, is then given by [6]:

$$
x_{i}(n)=\sum_{k=0}^{L-1} h_{i}(n) s(n-k)+u_{i}(n)
$$

In vector form, (1) can be written as:

$$
\begin{aligned}
& x_{i}(n) * h_{j}(n)=x_{j}(n) * h_{i}(n)+\theta_{i j} \\
& \theta_{i j}=u_{i}(n) * h_{j}(n)-u_{j}(n) * h_{i}(n)
\end{aligned}
$$

In order to construct measurement equation, the crossrelation equations in (2) is rewritten in matrix form as:

$$
\mathbf{C}(n) \mathbf{h}+\mathbf{v}_{2}(n)=0
$$

where $\mathbf{C}(n)=\left[\mathbf{x}_{2}(n),-\mathbf{x}_{1}(n)\right]$ represents measurement matrix, $\mathbf{x}_{i}(n)=\left[x_{i}(n), x_{i}(n-1), \cdots, x_{i}(n-L+1)\right]$. The state vector is denoted by $\mathbf{h}=\left[\mathbf{h}_{1}^{\mathbf{T}}, \mathbf{h}_{2}^{\mathbf{T}}\right]^{\mathbf{T}}$. The vector $\mathbf{v}_{1}(n)$ and the vector $\mathbf{v}_{2}(n)$ denote the process noise and measurement noise process, respectively.

The Kalman filter has two important equations (process and measurement equation) which are solved for a jointly optimal manner.

1) Process equation:

$$
\mathbf{h}(n+1)=\mathbf{F}(n+1, n) \mathbf{h}(n)+\mathbf{v}_{1}(n)
$$

2) Measurement equation:

$$
\mathrm{y}(n)=\mathbf{C}(n) \mathbf{h}(n)+\mathbf{v}_{2}(n)
$$

Table 1. Summary of the SKF algorithm.

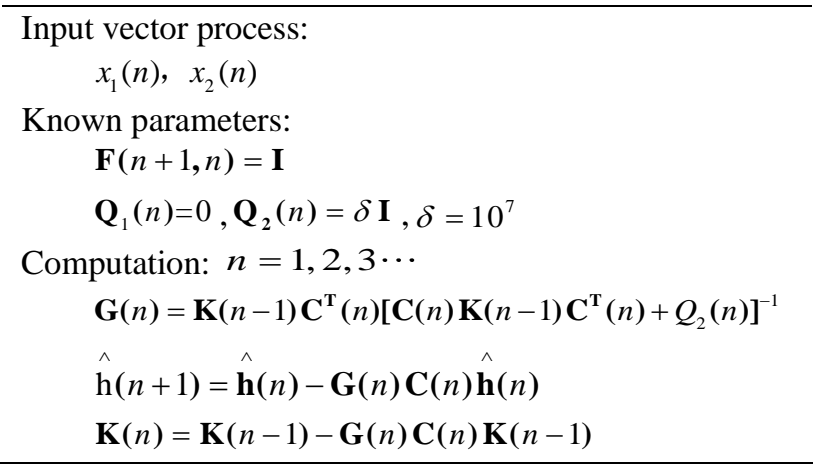

The cardiovascular system is a slow-changing system where $\mathbf{F}(n+1, n)=\mathbf{I}$ was assumed to be an identity matrix and it was also denoted to the state transition matrix. As shown in Table 1, for the simplified multichannel identification problem, the correlation matrix of process noise was assumed to $\mathbf{Q}_{1}(n)=0$ and the correlation matrix of measurement noise was assumed to $\mathbf{Q}_{2}(n)=\delta \mathbf{I}$. The matrix $\mathbf{G}(n)$ represented the Kalman gain matrix, $\mathbf{K}(n)=\mathbf{E}\left[\mathbf{e}(n) \mathbf{e}(n)^{\mathbf{T}}\right]$ represented the filter state-error correlation matrix and $\mathbf{e}(n)=\mathbf{h}(n)-\hat{\mathbf{h}}(n)$ represented the filter state-error vector.

\section{Results}

\subsection{Experimental data}

In the paper, we utilized a set of clinical data collected in a previous study. Invasive measurements of central aortic pressure waveforms were made at artery in 24 subjects undergoing cardiac surgery and the patients were written informed consent. The sampling rate of pulse data is 100 $\mathrm{Hz}$. In order to compare the proposed SKF algorithm with the CCA algorithm, measured $P_{a}$ waveforms were used for the input signal of the linear SIMO system to simulate corresponding $P_{b}$ waveforms and femoral artery pressure $\left(P_{f}\right)$ waveforms in the experiment.

As shown in Figure 2, measured $P_{a}$ waveforms were presented. At the same time, corresponding $P_{p}$ waveforms were simulated for a known two-channel FIR system that the order of FIR was assumed to be 10. The impulse responses of two-channel were given by

$$
\begin{aligned}
& h_{1}= {[0.7477,-0.4486,0.0093,0.0935,0.0841,} \\
&0.0187,0.0093,-0.0561,0.01869,0.3551] \\
& h_{2}= {[0.5357,0.2381,0.3571,02381,0.2381,} \\
&0.2143,0.0119,-0.0238,-0.0119,-0.9048]
\end{aligned}
$$

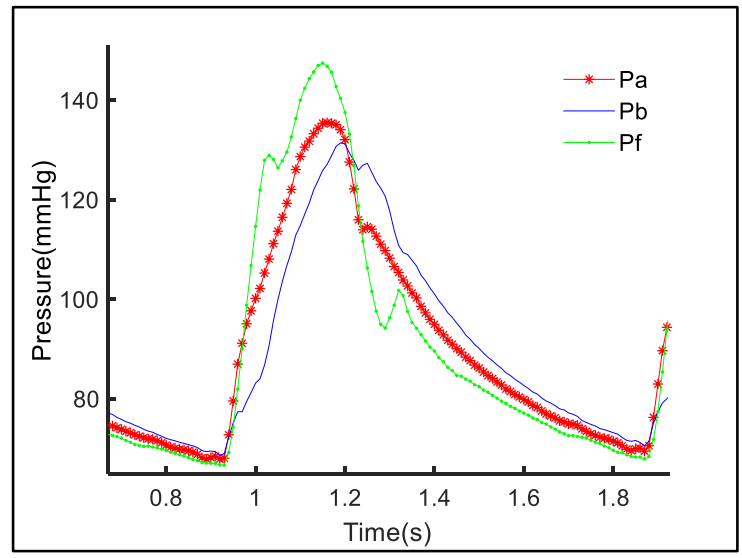

Figure 2. Sample of measured $P_{a}$ waveforms and corresponding $P_{p}$ waveforms.

\subsection{Evaluation index}


Root mean square error (RMSE) is used to calculate the difference between measured $P_{a}$ waveforms and estimated $P_{a}$ waveforms.

$$
R M S E=\sqrt{\frac{1}{N} \sum_{i=1}^{N}[\hat{s}(n)-s(n)]^{2}}
$$

Here, $\hat{s}(n)$ is the estimated input signal for the system identification, $s(n)$ is the measured input signal .

\subsection{Estimation of aortic pressure waveforms}

The distribution of the channel impulse response includes the zero point in Z-domain. Some studies have showed that upper limb and lower limb are enough different and have no common zero point. These channels are assumed to be linear and time invariant over each minute interval of analysis because of characterizes the dynamic properties of different arteries [7]. MBSI algorithms are usually very sensitive to observation noises. The SNR of the observed channel output signal can affect the convergence process. We investigated the performance of the proposed SKF and CCA algorithms for the different iterations and the different SNR in the constant length of the observed signal.

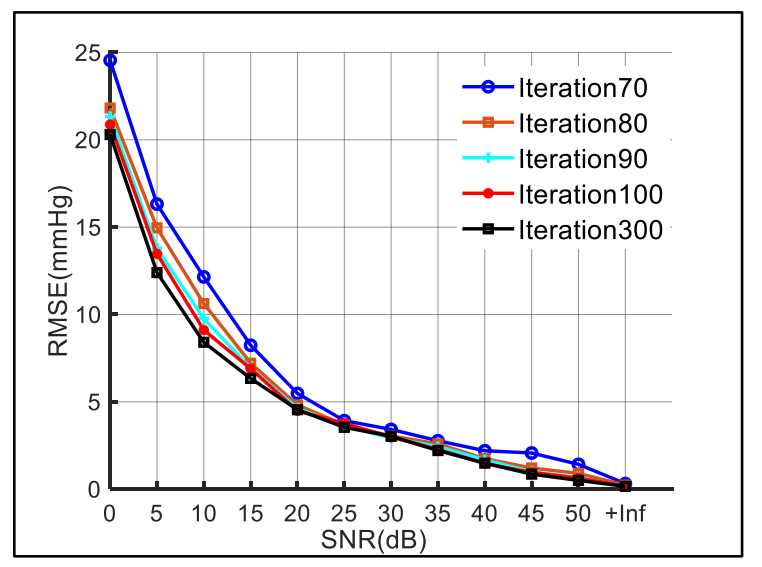

Figure 3. With the different iterations, average RMSE of measured and estimated $P_{a}$ waveforms using the SKF algorithm.

In this experiment, nonlinear aorta to peripheral artery system can be assumed to be linear for short time that the length of the output signal was set to 700. The influence of the number of iterations on $P_{a}$ waveforms estimation accuracy was investigated. It can be seen from Figure 3 that average RMSE decreased as the iteration times increased as expected. The iteration number was increased from 70 to 300 . If the iteration number was less than $70, P_{a}$ waveforms cannot be reconstructed. As shown in Figure 3, average RMSE was almost no longer decreased when the number of iterations was greater than or equal to 100 .
These results indicated that the proposed SKF algorithm had good overall performance when the number of iterations was more than 100 . In order to accurately and quickly estimate $P_{a}$ waveforms, the number of iterations of the SKF algorithm was set to 300. In the paper, the CCA algorithm [8] based on the linear SIMO system was applied as a benchmark to compare the performance of different blind system identification algorithms. It was reported that the SNR of $8 \%$ real pulse waveforms are more than $50 \mathrm{~dB}$ and the SNR of $1.5 \%$ real pulse waveforms are less than $10 \mathrm{~dB}[10]$. In the paper, we investigated that the SNR of pulse waveforms were increased from 0 to $50 \mathrm{~dB}$.

Table 2. The error metric obtained from measured and estimated $P_{a}$ waveforms using the CCA and SKF algorithms.

\begin{tabular}{ccccc}
\hline SNR & $\begin{array}{c}\text { CCA } \\
(\text { mean } \\
(\mathrm{mmHg}))\end{array}$ & $\begin{array}{c}\text { CCA } \\
(\mathrm{SD} \\
(\mathrm{mmHg}))\end{array}$ & $\begin{array}{c}\text { SKF } \\
(\text { mean } \\
(\mathrm{mmHg}))\end{array}$ & $\begin{array}{c}\text { SKF } \\
(\mathrm{SD} \\
(\mathrm{mmHg}))\end{array}$ \\
\hline 0 & 217.14 & 77.15 & 17.51 & 7.16 \\
5 & 76.29 & 27.39 & 12.09 & 5.15 \\
10 & 32.59 & 9.22 & 8.31 & 2.91 \\
15 & 15.87 & 5.50 & 6.20 & 2.40 \\
20 & 9.22 & 3.52 & 4.60 & 2.03 \\
$\mathbf{2 5}$ & $\mathbf{5 . 1 1}$ & $\mathbf{1 . 8 1}$ & $\mathbf{3 . 8 7}$ & $\mathbf{1 . 7 5}$ \\
30 & 4.27 & 2.06 & 2.98 & 1.59 \\
35 & 2.78 & 1.69 & 2.34 & 1.68 \\
40 & 1.68 & 1.19 & 1.57 & 1.13 \\
45 & 0.93 & 0.77 & 0.92 & 0.85 \\
50 & 0.47 & 0.59 & 0.48 & 0.53 \\
Inf & $\mathbf{0 . 2 8}$ & $\mathbf{0 . 4 9}$ & $\mathbf{0 . 2 7}$ & $\mathbf{0 . 5 1}$ \\
\hline
\end{tabular}

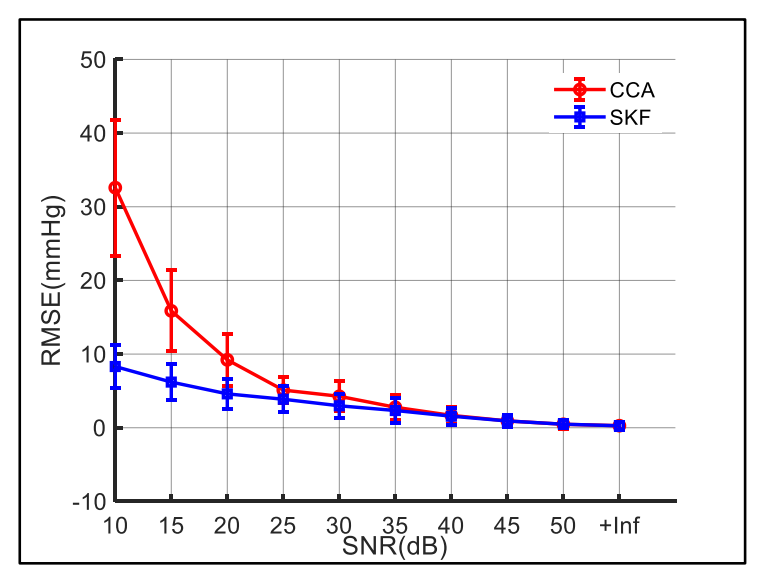

Figure 4. The error metric obtained from measured and estimated $P_{a}$ waveforms using the CCA and SKF algorithms (mean \pm SD).

As shown in Figure 4, the results of the proposed SKF algorithm showed an advantage over the CCA algorithm when the SNR was less than $25 \mathrm{~dB}$. However, the results of two methods almost had the same of performance when the 
SNR of the output signal was increased from $25 \mathrm{~dB}$ to $50 \mathrm{~dB}$. Table 2 clearly showed that measured and estimated $P_{a}$ waveforms using the CCA and SKF algorithms had good fitting in the noiseless scenario where the value of SNR is infinite. In the case of 24 sets of data, RMSE of measured and estimated $P_{a}$ waveforms using the CCA algorithm was about $0.28 \pm 0.49 \mathrm{mmHg}$. However, RMSE of measured and estimated $P_{a}$ waveforms using the SKF algorithm was about $0.27 \pm 0.51 \mathrm{mmHg}$.

In actual situations, noise is inevitable. Therefore, we used the proposed SKF and CCA algorithms to estimate $P_{a}$ waveforms when the SNR of $P_{p}$ waveforms were set to $25 \mathrm{~dB}$. Figure 5 gives an example of the comparison among the measured $P_{a}$ and the estimated $P_{a}$ using both the CCA and SKF algorithms, the results showed that RMSE of measured and estimated $P_{a}$ waveforms using the CCA algorithm was about $5.11 \pm 1.81 \mathrm{mmHg}$. However, measured and estimated $P_{a}$ waveforms using the SKF algorithm was about $3.87 \pm 1.75 \mathrm{mmHg}$.

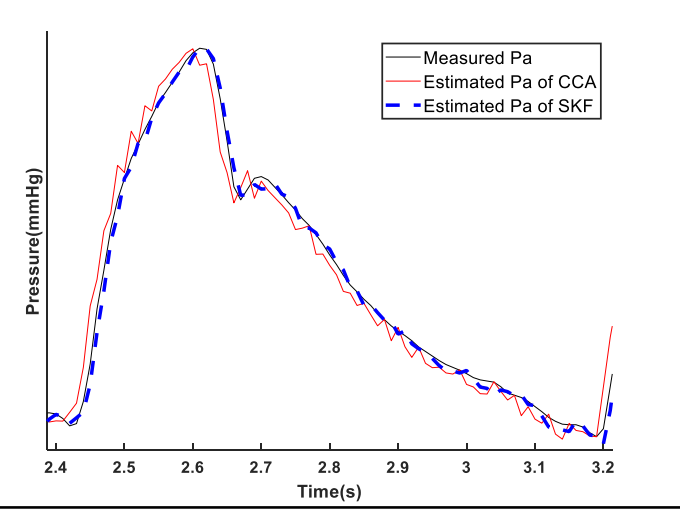

Figure 5. Measured and estimated $P_{a}$ waveforms using the CCA and SKF algorithms.

\section{Conclusion}

The results of simulation demonstrated that the performance of MBSI algorithms based on the proposed SKF algorithm was superior to the CCA algorithm over a wide range of SNR of the output signal. Generally, the property of the channel impulse response, the length of the observed output signal and the types of the source signal have a great influence on the convergence behavior of MBSI algorithms. The paper does not study these factors on the influence of blind system identification algorithms and the SNR of the observed channel output signal was only analyzed and discussed. In the future, these factors will be studied on the influence of MBSI algorithms. Our future work will use the data of animal experiments to verify the accuracy of the proposed SKF algorithm and accurately estimate $P_{a}$ waveforms which can be derived valuable clinical information to early diagnose cardiovascular disease.

\section{Acknowledgments}

The research reported here was, in part, supported by the National Natural Science Foundation of China (No. 61773110, No. 61374015), and the Fundamental Research Funds for the Central Universities (No. N161904002, No. N172008008). This work is also supported by the Open Program of Neusoft Research of Intelligent Healthcare Technology, Co. Ltd., Item number NRIHTOP1801.

\section{References}

[1] Suleman R, Padwal R, Hamilton P, et al, "Association between central blood pressure, arterial stiffness and mild cognitive impairment," Clinical Hypertension, vol. 23, no, 1, pp. 2, Jan. 2017.

[2] Cai T Y, Qasem A, Ayer J G, et al, "Central blood pressure in children and adolescents: non-invasive development and testing of novel transfer functions," Journal of Human Hypertension, vol. 31, no. 12, pp. 831-837, Aug. 2017.

[3] Hahn J O, Reisner A T, Asada H H, "Blind identification of two-channel IIR systems with application to central cardiovascular monitoring," Journal of Dynamic Systems Measurement \& Control, vol. 131, no. 5, pp. 545-553, Sep. 2009.

[4] Patel A, Li J, Finegan B, et al, "Aortic pressure estimation using blind identification approach on single input multiple output non-linear wiener systems," IEEE Transactions on Biomedical Engineering, vol. 65, no. 6, pp. 1193-1200, June. 2018.

[5] Abed M.K, et al, "Blind system identification," Proc. IEEE, vol. 85, no. 12, pp. 1310-1332, Sep. 1997.

[6] Mei, T, "Blind multichannel identification based on Kalman filter and eigenvalue decomposition," International Journal of Speech Technology, vol. 22, no. 1, pp. 1-11, Mar. 2019.

[7] Zhang Y, Asada H, "Blind system identification of noncoprime multichannel systems and its application to noninvasive cardiovascular monitoring," ASME Journal of Dynamic Systems: Measurement and Control, vol. 126, no. 4, pp. 834-847, Sep. 2004.

[8] Mayyala Q, Meraim K A, Zerguine A, "Structure-based subspace method for multi-channel blind system identification," IEEE Signal Processing Letters, vol. 24, no. 8, pp. 1183-1187, Feb. 2017.

[9] Huang Y A, Benesty J, “Adaptive multi-channel least mean square and Newton algorithms for blind channel identification," DBLP, vol. 82, no. 8, pp. 1127-1138, Aug. 2002.

[10] Xu L, Zhang $\mathrm{D}$, Wang $\mathrm{K}$, et al, "Baseline wander correction in pulse waveforms using wavelet-based cascaded adaptive filter," Computers in Biology and Medicine, vol. 37, no. 5, pp. 716-731, May. 2007.

Address for correspondence:

Lisheng $\mathrm{Xu}$.

Northeastern University, No. 195, Chuangxin Road, Hunnan District, Shen Yang City, Liaoning Province, China.

xuls@bmie.neu.edu.cn. 